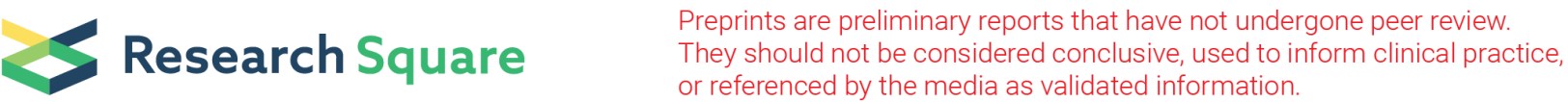

\section{Global Longitudinal Strain in Heart Transplantation Recipients Using Different Vendors: Reliability and Validity in a Tertiary Hospital in Colombia.}

Oscar Mauricio Perez Fernandez ( $\boldsymbol{\sigma}$ ompf@msn.com )

Fundacion Cardioinfantil Instituto de Cardiologia https://orcid.org/0000-0001-5868-2399

Hector M. Medina

Fundacion Cardioinfantil Instituto de Cardiologia

Mónica López

Fundacion Cardioinfantil Instituto de Cardiologia

Madeleine Barrera

Fundacion Cardioinfantil Instituto de Cardiologia

Azucena Martinez

Fundacion Cardioinfantil Instituto de Cardiologia

Jhonattan Benavides

Fundacion Cardioinfantil Instituto de Cardiologia

Juan C. Duran

Fundacion Cardioinfantil Instituto de Cardiologia

\section{Gabriel Salazar}

Fundacion Cardioinfantil Instituto de Cardiologia

Frida Tatiana Manrique

Fundacion Cardioinfantil Instituto de Cardiologia

\section{Research Article}

Keywords: Speckle-tracking strain, global longitudinal strain, inter-vendor, heart transplantation, agreement, left ventricle function.

Posted Date: June 1st, 2021

DOl: https://doi.org/10.21203/rs.3.rs-357760/v1

License: (c) (1) This work is licensed under a Creative Commons Attribution 4.0 International License.

Read Full License 


\section{Abstract}

Global Longitudinal Strain (GLS) is a useful tool to follow-up heart transplant (HT) recipients. Important inter-vendor variability of GLS measurements has been reported in healthy subjects and different conditions, but there is still limited evidence among HT patients. We assessed the reliability and validity of GLS using two vendors (General Electric and Philips) in a group of consecutive and stable adult HT recipients. Patients underwent two concurrent GLS analyses during their echocardiographic follow-up. We evaluated GLS inter-vendor reliability using Bland-Altman's limits of agreement (LOA) plots, computing its coverage probability (CP) and the intraclass correlation coefficient (ICC). Validity was assessed though receiver operating characteristics $(\mathrm{ROC})$ curves, predictive values, sensitivity and specificity of GLS for each vendor to detect a normal left ventricle function. 78 pairs of GLS studies in 53 stable HT patients were analyzed. We observed a modest inter-vendor reliability with a broad LOA (less than $50 \%$ of values falling out our CP of $2 \%$ and an ICC of 0.49 ). ROC analyses (areas under the curve of $0.824 \mathrm{Vs}$. 0.631 , $\mathrm{p}<0.05$ ) and diagnosis test indices (Sensitivity of 0.73 Vs. 0.64 ; and Specificity of 0.79 Vs. 0.50 ) favored GE over Philips. Inter-vendor variability for GLS analysis exceeded clinically acceptable limits in HT recipients. GLS from GE software seemed to show higher validity as compared to Philips'. The present study provides evidence to consider caution for the interpretation of GLS for clinical management in the follow-up of HT patients, especially when GLS is measured by different vendors.

\section{Introduction}

Heart Transplant (HT) recipients have special characteristics, including heart denervation, immunosuppressive therapy, exposure to allograft vasculopathy and cellular rejection, increasing the risk for myocardial dysfunction [1, 2]. Thus, the identification of early left ventricular dysfunction among HT patients has important therapeutic and prognostic implications.

Two-dimensional (2D) speckle-tracking echocardiography permits a reliable and comprehensive assessment of myocardial function. Global Longitudinal Strain (GLS) imaging can detect reduced myocardial function before any measurable changes in 2D echo parameters, such as left ventricle ejection fraction (LVEF) among HT patients [3-7] and other cardiovascular conditions[8, 9]. In fact, different studies and guidelines suggest the use of GLS as a predictor of acute cellular rejection (ACR) in HT recipients[3, 10-13].

Measuring GLS involves different sources of variability, such as ethnicity, image quality, tracking, operator experience and vendor choice[14], as demonstrated in healthy volunteers and different patient types [1517]. As a result, it is recommend that serial assessment of GLS for individual patients be performed under the same software plataform[18]. GLS are useful in different conditions such as infiltrative myocardiopathies[19], hypertrophic cardiomyopathy[20], cancer treatment related myocardiopathy[21], and valvular heart disease [22, 23], however inter- vendor GLS are reported in some of these conditions including cardiac amyloidosis and hypertrophic myocardiopathy (HCM)[24], ischemic cardiomyopathy[25] and chemotherapy related cardiotoxicity [26]. There is, however, limited data about 
inter-vendor GLS variability measurements in HT patients. Starting on 2005, our institution has performed over $100 \mathrm{HT}$. We thus sought to assess between-vendor GLS reliability and validity in a group of HT recipients using two widely available brands.

\section{Methods}

\section{Study sample and population}

This study included adult HT recipients in our institution who also attended subsequent their follow-up visits in our institution. We included consecutive stable adult HT recipients scheduled for a routine echocardiographic evaluation between November 2018 and April 2019, excluding cases with suboptimal echocardiographic image quality. Data regarding patient demographics, past medical history and transplantation procedures were retrieved from our program's records. The institutional review board at our institution approved the study protocol, in compliance with the ethical guidelines of the 1975 Declaration of Helsinki. All participants gave a written informed consent.

\section{Two-Dimensional Echocardiography}

All patients underwent first a comprehensive transthoracic echocardiogram (index echo) as clinically indicated. Four experienced, Cardiovascular Credentialing International certified sonographers performed additional echocardiographic. Images were obtained using any of the ultrasound systems from two vendors: Vivid E9 (General Electric -GE-) and EPIQ 7 or iE33 (Philips). Sequentially, two-dimensional images required for GLS analysis were obtained by the same sonographer in the second vendor. The vendors associated with this analysis did not have a role in the design, data collection or interpretation of this study.

\section{Speckle-tracking Strain Analysis}

An experienced observer performed two-dimensional speckle-tracking analysis blinded to clinical data, using two vendor-specific software packages: EchoPAC PC version 110.1.2 (GE) and QLAB versions 10.0 (Philips). GLS was obtained from frame-by-frame tracking of speckle patterns throughout the left ventricle (LV) myocardium in standard 2D cine loops. For optimal tracking results, we allowed manual adjustment of the speckle area of interest. GLS measures were done at the time of image acquisition for each vendor. Poor image acquisition or artifacts were excluded from analysis. We report the absolute values of GLS in the text, figures and tables, avoiding negative values of GLS. Any repeated measurement of GLS within the same patient was blinded to previous analysis (informed consent in each visit was mandatory).

\section{Statistical Analysis}

Data are presented as means \pm standard deviation (SD), medians (interquartile range), or absolute numbers (percentages). Normal distribution assumptions were tested with the Shapiro-Wilk statistic and 
the Kolmogorov-Smirnov test. A two-tailed $P$ value $<.05$ was assumed as statistically significant in all comparative analyses.

We evaluated GLS inter-vendor reliability using different methods. First, Bland-Altman plots were constructed by computing limits of agreement (LOA) ranges ( \pm 1.96 standard deviation of the differences between the 2 imaging modalities). Approximately $95 \%$ of the differences are expected to fall within this range [27], where agreement is proportional to the width of the LOA between the measured values. Second, we calculated the coverage probability $[27,28]$, which is the proportion of values within the prespecified acceptable paired absolute difference. For GLS, we pre-specified paired absolute difference of $2 \%$ as a clinically acceptable boundary. Third, we used the intraclass correlation coefficient (ICC).

Our validity assessment was based on GLS's diagnostic performance of each vendor. It started by generating receiver operating characteristics (ROC) curves, setting a normal LEVF ( $\geq 55 \%)$ as dependent variable. We computed vendor-specific area under the curve (AUC) in order to identify the cut-off points with the best diagnostic yield. We obtained sensitivity, specificity, positive predictive value (PPV), negative predictive values (NPV), as well as likelihood ratios (LRs) for each vendor.

Statistical analyses were performed using the Statistical Package for the Social Sciences (SPSS) version 24.0 (SPSS, Inc, Chicago, IL), Epidat software (version 4.2) and Stata (version 13.0, StataCorp). We report the above estimates along with their $95 \%$ confidence intervals $(95 \% \mathrm{Cl})$ and considered a $P$ value $<.05$ as statistically significant for hypothesis testing.

\section{Results}

\section{Clinical and echocardiographic findings}

We included a total of 78 prospective and consecutive pairs of echocardiographic studies in 53 stable HT patients. The baseline characteristics of the study participants are presented in Table 1. Patients were mainly male (84.9\%) with a mean age of $51.6 \pm 13.9$ years. The median follow-up time after HT was 77.9 (IQR: 41.0 - 110.4) months. The more common causes for HT were idiopathic, ischemic and Chagas' chronic cardiomyopathy. The index echocardiogram showed a mean LVEF of $55.6 \pm 7.6 \%$ and LV volumes were within normal limits.

Only $44(56.4 \%)$ studies reported a normal LVEF ( $\geq 55 \%)$. GLS values generated for both Philips and GE software packages discriminated differences between patients with normal Vs. reduced LVEF (Figure 1, Table 2). GE's GLS mean values were numerically, but not statistically lower than Philips' in the low LVEF group $(12.85 \pm 3.7 \%$ vs $14.31 \pm 4.1 \%$, respectively, $p=0.128)$.

\section{Inter-vendor reliability of GLS}

As expected, mean GLS was similar for both vendors $(15.41 \pm 3.62 \%$ and $15.03 \pm 3.55 \%$ for Philips and $\mathrm{GE}$, respectively). However, we found broad LOA between vendors, ranging from $-6.72 \%$ to $7.49 \%$. Only $46.2 \%$ of values fell within our pre-defined coverage probability of $2 \%$, as highlighted in the Bland-Altman 
Plot of Figure 2. An intraclass correlation coefficient (ICC) of 0.49 demonstrated only a moderate reliability. The results of all indices comparing GLS for both vendors are summarized in Table 3. A fourchamber frame with left ventricle bullseye displaying segmental peak strain and GLS values as well as the corresponding polar map for each vendor for two different patients (agreement and disagreement) are presented in Figure 3.

\section{Validity analysis}

ROC curves and AUC (Figure 4) showed a better, and statistically significant ( $p=0.022)$ performance of GE's values, as compared to Philips'. Best GLS cut-off points were $15.4 \%$ for GE and $14.7 \%$ for Philips, respectively. GE's values showed higher sensitivity, specificity, PPV, NPV, LR+ and a lower LR- (Table 4).

\section{Impact of ACR on GLS}

Data about the grade of previous ACR were not available. We conducted an exploratory analysis to determine the impact of previous ACR events $(0,1$ or $\geq 2)$ on GLS. Despite a numerically inverse association between GLS and number of ACR in both providers (GE and Philips), we observed no significant association (Supplementary Table 1 and Supplementary Figures 1 and 2).

\section{Discussion}

\section{Main Findings}

In this study, we performed simultaneous echocardiographic assessments for GLS analyses in a group of stable HT patients, aiming to evaluate inter-vendor GLS reliability and validity. Our findings do not support that data obtained with the two GLS software packages under investigation could be clinically exchangeable. The inter-vendor LOA were broad (-6.72\% to $7.49 \%)$, with less than half of measurements falling into our clinically acceptable boundary of $2 \%$ difference. Also, our ICC estimate indicating only a moderate reliability. Although GLS data from both vendors discriminate LVEF categories, in this study the GE platform showed a better diagnostic performance, suggesting higher validity for GLS evaluation in HT recipients. Neither brand identified an association between GLS and the number of previous ACR events.

\section{Variability of GLS among healthy individuals and different clinical conditions}

Based on a multicenter international study including more than 2000 individuals [29], the World Alliance Societies of Echocardiography (WASE) proposed the normal values for GLS ( $17 \%$ to $24 \%$ for males and $18 \%$ to $26 \%$ for females). Different studies have reported, however, inter-vendor GLS variability in healthy volunteers. For example, a previous study[30], reported LOA between -3.41 to 1.81 comparing Philips Vs. GE with an inter-vendor bias of -0.8 and normal mean GLS of $17.88 \pm 1.84$ and $18.69 \pm 1.72$, respectively. Similar results in that study were reported for vendor-independent software (TOMTEC and Epsilon). Other studies in healthy volunteers showed a wide LOA ranging from -6.0 up to 9.0 [31-33]. 
Reports of GLS in patients with different heart diseases have shown similarly concerning results. In ischemic cardiomyopathy, wide inter-vendor variability have been reported not only for GLS[25,34], but also for segmental longitudinal strain and layer-specific longitudinal strain [35], even in cancer patients[26]. Another study including subjects with amyloidosis and hypertrophic myocardiopathy with simultaneous image acquisitions using GE, Siemens and Philips machines, found global and regional between-vendors variations in longitudinal strain [24]. As in our study, the Philips software tended to report higher GLS values, as compared with other brands (GE and VVI) among patients with cardiac amyloidosis. Although authors of that study did not report their LOA, their Bland-Altman plots showed wide amplitude of distribution, more evident for the Siemens Vs. Philips plot. We did not include other vendors in our study, because of our echocardiography lab only uses GE and Philips.

\section{GLS in HT recipients}

Although still subject of controversy, studies have shown GLS as an important marker for future LV dysfunction and ACR after HT [5, 36-38]. Inter-vendor reproducibility in diagnosis performance of ACR has been reported as low, with inconsistent results (between Siemens and TOMTEC) [6]. Our study did not specifically aim at detecting such association. However, in the exploratory analysis we found an inverse, but no significant relationship between the number of previous ACR and GLS in any vendor.

Different studies in HT patients[12, 13,39] reported low GLS values, despite a mean normal LVEF, as we do in this study. It is known the higher capacity of GLS to detect myocardial deformation in contrast to LVEF (that shows only LV volumetric changes based on Simpson's method). However, controversy still exists on when GLS values are abnormal in some myocardial diseases with thickened walls or small LV cavity (HCM, amyloidosis) despite a preserved LVEF[9]. The relatively low GLS values we found in HT recipients would represent normality or be the initial step for myocardial dysfunction.

Guidelines recommend measuring GLS in HT patients as a suitable parameter to diagnose subclinical allograft dysfunction, and comparing changes occurring during serial evaluations[10]. Our study shows that mean GLS in HT patients with normal LVEF were within normal limits, and similar for both vendors (16.27 \pm 3.0 for Philips Vs. $16.72 \pm 2.3$ for GE). As expected, among HT patients with low LVEF, mean GLS were significantly lower as compared to those with normal LVEF with consistency between the two vendors assessed (14.31 \pm 4.1 for Philips Vs. $12.85 \pm 3.7$ for GE, respectively).

\section{Reliability and Validity of GLS in HT recipients}

To our knowledge, this is the first study evaluating inter-vendor GLS variability among HT patients. We found, as previously reported in healthy subjects and patients with different heart diseases, a wide LOA between vendors. Based on a previous study by Pellikka et al[28], we used a coverage probability as a threshold for acceptable reliability. We found that less than half of pairs of measures are into $2 \%$ of intervendor difference. Although the study of Pellikka et al was done to compare agreement of LVEF between echo and cardiac magnetic resonance, we extrapolated the concept to GLS. This finding was consistent with an ICC of 0.49 , demonstrating a moderate inter-vendor agreement. Based on such differences, our 
findings suggest that GE's GLS performs better than Philips'. Also, our results enhance the concept that GLS have multiple sources of variability that should be considered in clinical management and future research.

\section{Limitations}

Our study has a number of limitations. First, these results are from a single center with a relatively small sample size. However, we allowed paired, repeated measurements in the same patients over time increasing our sample for analyses. Second, we only included two vendor-specific software packages. There is, however, a wide spectrum of software (both independent and vendor-specific) available now, with significant differences already reported[30-33]. The two vendors included in our analyses are heavily used, and available worldwide, making our results applicable to a many echocardiographic laboratories using the same providers. However, this study has strengths to know: our HT patients were heterogeneous with a different follow-up time since HT and our echocardiographic studies were simultaneously obtained to reducing this risk of biases. However, it is possible that not-measured variables may alter the results presented here.

\section{Conclusion}

Our study demonstrates a wide inter-vendor GLS variability with roughly half of the values exceeding a clinically acceptable limits of agreement among HT recipients in our institution. GLS computed by GE software seemed to be more associated with clinical characteristics, as compared to Philips in this population. These data provide evidence to consider caution for the use of different GLS computations for clinical management and decision making in the follow-up of HT patients. On the light of our results, we recommend to use the same software platform for GLS analysis in HT recipients. Further studies are needed to support the results presented in this study.

\section{Declarations}

Funding: This research did not receive any specific grant from funding agencies in the public, commercial, or not-for-profit sectors.

Conflict of interest: The authors declare that they have no conflict of interest.

Ethical approval: all procedures were in accordance with the ethical standards of the institutional research committee and with the Helsinki declaration and its later amendments.

Informed consent: Written informed consent was obtained from the subjects prior to participation in the study.

\section{Author contributions}


Oscar Mauricio Perez-Fernandez: Conceptualization, Methodology, Validation, Formal analysis, Writing Original draft. Hector M. Medina: Investigation, Writing - Review and Editing. Juan C. Villar: Methodology, Formal analysis, Writing - Review and Editing.

Mónica Lopez: Resources. Madeleine Barrera: Conceptualization, Investigation. Azucena Martinez: Conceptualization, Investigation. Jhonattan Benavides: Conceptualization. Juan C. Duran: Investigation. Gabriel Salazar: Supervision, Project administration. Frida Tatiana Manrique: Conceptualization, Writing - Review and Editing, Supervision, Project administration.

Acknowledgments: we acknowledge the valuable contribution of all Sonographers (Isabel Zorro, Cristina Villamizar, and Gonzalo Flórez) for the technical support and image acquisitions during the development of this study. The authors would like to thank Elizabeth Cruz Tapias for her help in designing the figures.

\section{References}

1. Cotts WG, Oren RM (1997) Function of the Transplanted Heart: Unique Physiology and Therapeutic Implications. Am J Med Sci 314:164-172. https://doi.org/10.1016/S0002-9629(15)40190-9

2. Söderlund C, Rådegran G (2015) Immunosuppressive therapies after heart transplantation--The balance between under- and over-immunosuppression. Transplant Rev (Orlando) 29:181-9. https://doi.org/10.1016/j.trre.2015.02.005

3. Elkaryoni A, Altibi AM, Khan MS, et al (2020) Global longitudinal strain assessment of the left ventricle by speckle tracking echocardiography detects acute cellular rejection in orthotopic heart transplant recipients: A systematic review and meta-analysis. Echocardiography 37:302-309. https://doi.org/10.1111/echo.14586

4. Marciniak A, Eroglu E, Marciniak M, et al (2007) The potential clinical role of ultrasonic strain and strain rate imaging in diagnosing acute rejection after heart transplantation. Eur $\mathrm{J}$ Echocardiogr 8:213-21. https://doi.org/10.1016/j.euje.2006.03.014

5. Mingo-Santos S, Moñivas-Palomero V, Garcia-Lunar I, et al (2015) Usefulness of Two-Dimensional Strain Parameters to Diagnose Acute Rejection after Heart Transplantation. J Am Soc Echocardiogr 28:1149-56. https://doi.org/10.1016/j.echo.2015.06.005

6. Ruiz-Ortiz M, Rodriguez-Diego S, Delgado M, et al (2019) Myocardial deformation and acute cellular rejection after heart transplantation: Impact of inter-vendor variability in diagnostic effectiveness. Echocardiography 36:2185-2194. https://doi.org/10.1111/echo.14544

7. Saleh HK, Villarraga HR, Kane GC, et al (2011) Normal left ventricular mechanical function and synchrony values by speckle-tracking echocardiography in the transplanted heart with normal ejection fraction. J Hear Lung Transplant 30:652-658. https://doi.org/10.1016/j.healun.2010.12.004

8. Koyama J, Ray-Sequin PA, Falk RH (2003) Longitudinal myocardial function assessed by tissue velocity, strain, and strain rate tissue Doppler echocardiography in patients with AL (primary) cardiac amyloidosis. Circulation 107:2446-52. https://doi.org/10.1161/01.CIR.0000068313.67758.4F 
9. Luis SA, Chan J, Pellikka PA (2019) Echocardiographic Assessment of Left Ventricular Systolic Function: An Overview of Contemporary Techniques, Including Speckle-Tracking Echocardiography. Mayo Clin Proc 94:125-138. https://doi.org/10.1016/j.mayocp.2018.07.017

10. Badano LP, Miglioranza MH, Edvardsen T, et al (2015) European Association of Cardiovascular Imaging/Cardiovascular Imaging Department of the Brazilian Society of Cardiology recommendations for the use of cardiac imaging to assess and follow patients after heart transplantation. Eur Hear journal Cardiovasc Imaging 16:919-48. https://doi.org/10.1093/ehjci/jev139

11. Syeda B, Höfer P, Pichler P, et al (2011) Two-dimensional speckle-tracking strain echocardiography in long-term heart transplant patients: A study comparing deformation parameters and ejection fraction derived from echocardiography and multislice computed tomography. Eur J Echocardiogr 12:490496. https://doi.org/10.1093/ejechocard/jer064

12. Saleh HK, Villarraga HR, Kane GC, et al (2011) Normal left ventricular mechanical function and synchrony values by speckle-tracking echocardiography in the transplanted heart with normal ejection fraction. J Hear Lung Transplant 30:652-658. https://doi.org/10.1016/j.healun.2010.12.004

13. Pichler $P$, Binder $T$, Höfer $P$, et al (2012) Two-dimensional speckle tracking echocardiography in heart transplant patients: Three-year follow-up of deformation parameters and ejection fraction derived from transthoracic echocardiography. Eur Heart J Cardiovasc Imaging 13:181-186.

https://doi.org/10.1093/ejechocard/jer239

14. Collier P, Phelan D, Klein A (2017) A Test in Context: Myocardial Strain Measured by Speckle-Tracking Echocardiography. J. Am. Coll. Cardiol. 69:1043-1056

15. Castel AL, Szymanski C, Delelis F, et al (2014) Prospective comparison of speckle tracking longitudinal bidimensional strain between two vendors. Arch Cardiovasc Dis 107:96-104. https://doi.org/10.1016/j.acvd.2014.01.007

16. Farsalinos KE, Daraban AM, Ünlü S, et al (2015) Head-to-Head Comparison of Global Longitudinal Strain Measurements among Nine Different Vendors. J Am Soc Echocardiogr 28:1171-1181.e2. https://doi.org/10.1016/j.echo.2015.06.011

17. Hoogslag GE, Abou R, Joyce E, et al (2015) Comparison of Changes in Global Longitudinal Peak Systolic Strain After ST-Segment Elevation Myocardial Infarction in Patients With Versus Without Diabetes Mellitus. Am J Cardiol 116:1334-9. https://doi.org/10.1016/j.amjcard.2015.07.061

18. Lang RM, Badano LP, Mor-Avi V, et al (2015) Recommendations for Cardiac Chamber Quantification by Echocardiography in Adults: An Update from the American Society of Echocardiography and the European Association of Cardiovascular Imaging. J Am Soc Echocardiogr 28:1-39.e14. https://doi.org/10.1016/j.echo.2014.10.003

19. Agha AM, Parwani P, Guha A, et al (2018) Role of cardiovascular imaging for the diagnosis and prognosis of cardiac amyloidosis. Open Hear 5:e000881. https://doi.org/10.1136/openhrt-2018000881 
20. Tower-Rader A, Mohananey D, To A, et al (2019) Prognostic Value of Global Longitudinal Strain in Hypertrophic Cardiomyopathy. JACC Cardiovasc Imaging 12:1930-1942. https://doi.org/10.1016/j.jcmg.2018.07.016

21. Santoro C, Esposito R, Lembo M, et al (2019) Strain-oriented strategy for guiding cardioprotection initiation of breast cancer patients experiencing cardiac dysfunction. Eur Hear journal Cardiovasc Imaging 20:1345-1352. https://doi.org/10.1093/ehjci/jez194

22. Santoro C, Galderisi M, Esposito R, et al (2019) Global longitudinal strain is a hallmark of cardiac damage in mitral regurgitation: the Italian arm of the European Registry of mitral regurgitation (EuMiClip). Cardiovasc Ultrasound 17:28. https://doi.org/10.1186/s12947-019-0178-7

23. Dahl JS, Videbæk L, Poulsen MK, et al (2012) Global Strain in Severe Aortic Valve Stenosis. Circ Cardiovasc Imaging 5:613-620. https://doi.org/10.1161/CIRCIMAGING.112.973834

24. Sperry BW, Sato K, Phelan D, et al (2019) Regional Variability in Longitudinal Strain Across Vendors in Patients With Cardiomyopathy Due to Increased Left Ventricular Wall Thickness. Circ Cardiovasc Imaging 12:. https://doi.org/10.1161/CIRCIMAGING.119.008973

25. Badano LP, Cucchini U, Muraru D, et al (2013) Use of three-dimensional speckle tracking to assess left ventricular myocardial mechanics: inter-vendor consistency and reproducibility of strain measurements. Eur Hear journal Cardiovasc Imaging 14:285-93.

https://doi.org/10.1093/ehjci/jes184

26. Ancedy Y, Ederhy S, Lang S, et al (2018) Multilayer global longitudinal strain in patients with cancer: A comparison of two vendors. Arch Cardiovasc Dis 111:285-296. https://doi.org/10.1016/j.acvd.2017.11.003

27. Barnhart HX, Yow E, Crowley AL, et al (2016) Choice of agreement indices for assessing and improving measurement reproducibility in a core laboratory setting. Stat Methods Med Res 25:29392958. https://doi.org/10.1177/0962280214534651

28. Pellikka PA, She L, Holly TA, et al (2018) Variability in Ejection Fraction Measured By Echocardiography, Gated Single-Photon Emission Computed Tomography, and Cardiac Magnetic Resonance in Patients With Coronary Artery Disease and Left Ventricular Dysfunction. JAMA Netw open 1:e181456. https://doi.org/10.1001/jamanetworkopen.2018.1456

29. Asch FM, Miyoshi T, Addetia K, et al (2019) Similarities and Differences in Left Ventricular Size and Function among Races and Nationalities: Results of the World Alliance Societies of Echocardiography Normal Values Study. J Am Soc Echocardiogr 32:1396-1406.e2. https://doi.org/10.1016/j.echo.2019.08.012

30. Nagata Y, Takeuchi M, Mizukoshi K, et al (2015) Intervendor Variability of Two-Dimensional Strain Using Vendor-Specific and Vendor-Independent Software. J Am Soc Echocardiogr 28:630-641. https://doi.org/10.1016/j.echo.2015.01.021

31. Takigiku K, Takeuchi M, Izumi C, et al (2012) Normal Range of Left Ventricular 2-Dimensional Strain. Circ J 76:2623-2632. https://doi.org/10.1253/circj.CJ-12-0264 
32. Biaggi P, Carasso S, Garceau P, et al (2011) Comparison of Two Different Speckle Tracking Software Systems: Does the Method Matter? Echocardiography 28:539-547. https://doi.org/10.1111/j.15408175.2011.01386.x

33. Manovel A, Dawson D, Smith B, Nihoyannopoulos $P$ (2010) Assessment of left ventricular function by different speckle-tracking software. Eur J Echocardiogr 11:417-421. https://doi.org/10.1093/ejechocard/jep226

34. Mirea O, Pagourelias ED, Duchenne J, et al (2018) Variability and Reproducibility of Segmental Longitudinal Strain Measurement. JACC Cardiovasc Imaging 11:15-24. https://doi.org/10.1016/j.jcmg.2017.01.027

35. Ünlü S, Mirea O, Pagourelias ED, et al (2019) Layer-Specific Segmental Longitudinal Strain Measurements: Capability of Detecting Myocardial Scar and Differences in Feasibility, Accuracy, and Reproducibility, Among Four Vendors A Report From the EACVI-ASE Strain Standardization Task Force. J Am Soc Echocardiogr 32:624-632.e11. https://doi.org/10.1016/j.echo.2019.01.010

36. Tseng AS, Gorsi US, Barros-Gomes S, et al (2018) Use of speckle-tracking echocardiography-derived strain and systolic strain rate measurements to predict rejection in transplant hearts with preserved ejection fraction. BMC Cardiovasc Disord 18:241. https://doi.org/10.1186/s12872-018-0980-4

37. Clemmensen TS, Løgstrup BB, Eiskjær H, Poulsen SH (2015) Changes in Longitudinal Myocardial Deformation during Acute Cardiac Rejection: The Clinical Role of Two-Dimensional Speckle-Tracking Echocardiography. J Am Soc Echocardiogr 28:330-339. https://doi.org/10.1016/j.echo.2014.10.015

38. Antończyk K, Niklewski T, Antończyk R, et al (2018) Speckle-Tracking Echocardiography for Monitoring Acute Rejection in Transplanted Heart. Transplant Proc 50:2090-2094. https://doi.org/10.1016/j.transproceed.2018.03.112

39. Syeda B, Höfer P, Pichler P, et al (2011) Two-dimensional speckle-tracking strain echocardiography in long-term heart transplant patients: a study comparing deformation parameters and ejection fraction derived from echocardiography and multislice computed tomography. Eur J Echocardiogr 12:490-6. https://doi.org/10.1093/ejechocard/jer064

\section{Tables}

Table 1 Clinical characteristics of patients with Heart Transplantation. 


\begin{tabular}{|c|c|}
\hline \multicolumn{2}{|l|}{ Characteristics $(n=53)$} \\
\hline Age $(y)$ & $51.6 \pm 13.9$ \\
\hline Male & $45(84.9 \%)$ \\
\hline Follow-up, months since HT & $77.9(41.0-110.4)$ \\
\hline \multicolumn{2}{|l|}{ Transplant indication* } \\
\hline Ischemic cardiomyopathy & 16 \\
\hline Idiopathic & 15 \\
\hline Chagas disease & 11 \\
\hline Myocarditis & 3 \\
\hline Toxic & 1 \\
\hline Family pattern & 1 \\
\hline Arrhythmia & 1 \\
\hline Lymphoma & 1 \\
\hline Arrhythmogenic cardiomyopathy & 1 \\
\hline Ebstein anomaly & 1 \\
\hline Non-compacted myocardiopathy & 1 \\
\hline Post-partum & 1 \\
\hline \multicolumn{2}{|l|}{ Comorbidities } \\
\hline Dyslipidemia & $31(58.5 \%)$ \\
\hline Hypothyroidism & $18(34.0 \%)$ \\
\hline Hypertension & $13(24.5)$ \\
\hline Chagas disease & $11(20.8)$ \\
\hline Diabetes mellitus & $11(20.8)$ \\
\hline Chronic kidney disease & $10(18.9)$ \\
\hline Chagas reactivation & $8(15.1)$ \\
\hline Thrombophilia & $6(11.3)$ \\
\hline Cancer & $5(9.4)$ \\
\hline Stroke & $5(9.4)$ \\
\hline Epilepsy & $3(5.7)$ \\
\hline
\end{tabular}

Page 12/18 


\begin{tabular}{|ll|}
\hline ICD & $3(5.7)$ \\
\hline Hepatitis B & $1(1.9)$ \\
\hline HIV infection & $1(1.9)$ \\
\hline Echocardiographic findings $^{\dagger}$ & \\
\hline LVEF $(\%)$ & $55.6 \pm 7.6$ \\
\hline LV EDV $(\mathrm{mL})$ & $72.2 \pm 22.4$ \\
\hline LV EDV $\left(\mathrm{mL} / \mathrm{m}^{2}\right)$ & $39.9 \pm 10.4$ \\
\hline LV ESV $(\mathrm{mL})$ & $32.9 \pm 12.3$ \\
\hline LV ESV $\left(\mathrm{ml} / \mathrm{m}^{2}\right)$ & $18.1 \pm 5.6$ \\
\hline
\end{tabular}

EDV, end-diastolic volume; EF, ejection fraction; ESV, end-systolic volume; LV, left ventricle; $H T$, heart transplant; ICD, implantable cardio-defibrillator; HIV, human immunodeficiency virus.

Data are expressed as mean $\pm \mathrm{SD}$, median (IQR) or as number (percentage).

*Data are expressed as frequencies.

${ }^{\dagger}$ Data correspond to index echocardiogram (any of the 2 vendors).

Table 2 GLS values according to $\operatorname{LVEF}^{\dagger}$ ( $\geq 55 \%$ vs $<55 \%$ ) for the two vendors assessed.

\begin{tabular}{|lllll|}
\hline & Philips & \multicolumn{3}{c|}{ GE } \\
\cline { 2 - 5 } & LVEF $\geq \mathbf{5 5 \%}$ & LVEF $<\mathbf{5 5 \%}$ & LVEF $\geq \mathbf{5 5 \%}$ & LVEF $<\mathbf{5 5 \%}$ \\
& $\mathbf{n}=\mathbf{4 4}$ & $\mathbf{n = 3 4}$ & $\mathbf{n}=\mathbf{4 4}$ & $\mathbf{n = 3 4}$ \\
\hline GLS, mean \pm SD & $16.27 \pm 3.0$ & $14.31 \pm 4.1$ & $16.72 \pm 2.3$ & $12.85 \pm 3.7$ \\
\hline GLS mean difference* & $1.95(0.35,3.55)$ & $3.87(2.50,5.23)$ \\
\hline p value & 0.017 & & $<0.001$ & \\
\hline
\end{tabular}

LVEF, left ventricle ejection fraction; GLS, global longitudinal strain; $\mathrm{Cl}$, confidence interval

*Represented as mean difference and lower and upper limits of $95 \% \mathrm{Cl}$.

tData correspond to index echocardiogram (any of the 2 vendors).

Paired $t$ test for LVEF $\geq 55 \%$ vs LVEF $<55 \%$ groups. 
Table 3 Variability of GLS values by vendor-specific software.

\begin{tabular}{|ll|}
\hline GLS Philips & $15.41 \pm 3.62$ \\
\hline GLS GE & $15.03 \pm 3.55$ \\
\hline Bland-Altman LOA* & $-6.72 \%, 7.49 \%$ \\
\hline Coverage probability+(\%), 95\% Cl & $46.2 \%(34.6,57.7)$ \\
\hline ICC, 95\% Cl & $0.490(0.302-0.463)$ \\
\hline
\end{tabular}

ICC, intraclass correlation coefficient; GLS, global longitudinal strain; LOA, limits of agreement; $\mathrm{Cl}$, confidence interval

Data are expressed as mean \pm SD

*Bland-Altman LOA defined by \pm 1.96 standard deviation of the differences between the 2 imaging modalities. Approximately $95 \%$ of the differences are expected to fall within this range.

+Coverage probability is the proportion of participants who fall within the pre-specified acceptable paired absolute difference. For GLS, the pre-specified acceptable paired absolute difference was $2 \%$ Data are reported as a proportion and its $95 \% \mathrm{Cl}$.

Table 4 Diagnostic performance of GLS values ${ }^{\ddagger}$ obtained from the two vendors assessed to discriminate normal LVEF.

\begin{tabular}{|lll|}
\hline & Philips & GE \\
\hline AUC* & $0.63(0.50,0.76)$ & $0.82(0.723,0.93)$ \\
\hline Sensitivity & $0.64(0.48,0.77)$ & $0.73(0.57,0.85)$ \\
\hline Specificity & $0.50(0.33,0.67)$ & $0.79(0.62,0.91)$ \\
\hline PPV & $0.62(0.47,0.76)$ & $0.82(0.66,0.92)$ \\
\hline NPV & $0.52(0.34,0.69)$ & $0.69(0.52,0.82)$ \\
\hline LR + & $1.27(0.85,1.91)$ & $3.53(1.78,7.00)$ \\
\hline LR - & $0.73(0.43,1.22)$ & $0.34(0.21,0.57)$ \\
\hline
\end{tabular}

AUC, area under the curve; $\mathrm{Cl}$, confidence interval; NPV, negative predictive value, LR, likelihood ratio; PPV, positive predictive value.

${ }^{¥}$ Cut-off point of GLS to calculate parameters were $14.7 \%$ and $15.4 \%$ for Philips and GE, respectively. 
Data are expressed as a parameter value and lower and upper limits of $95 \% \mathrm{Cl}$.

${ }^{*} \mathrm{p}=0.022$

Figures
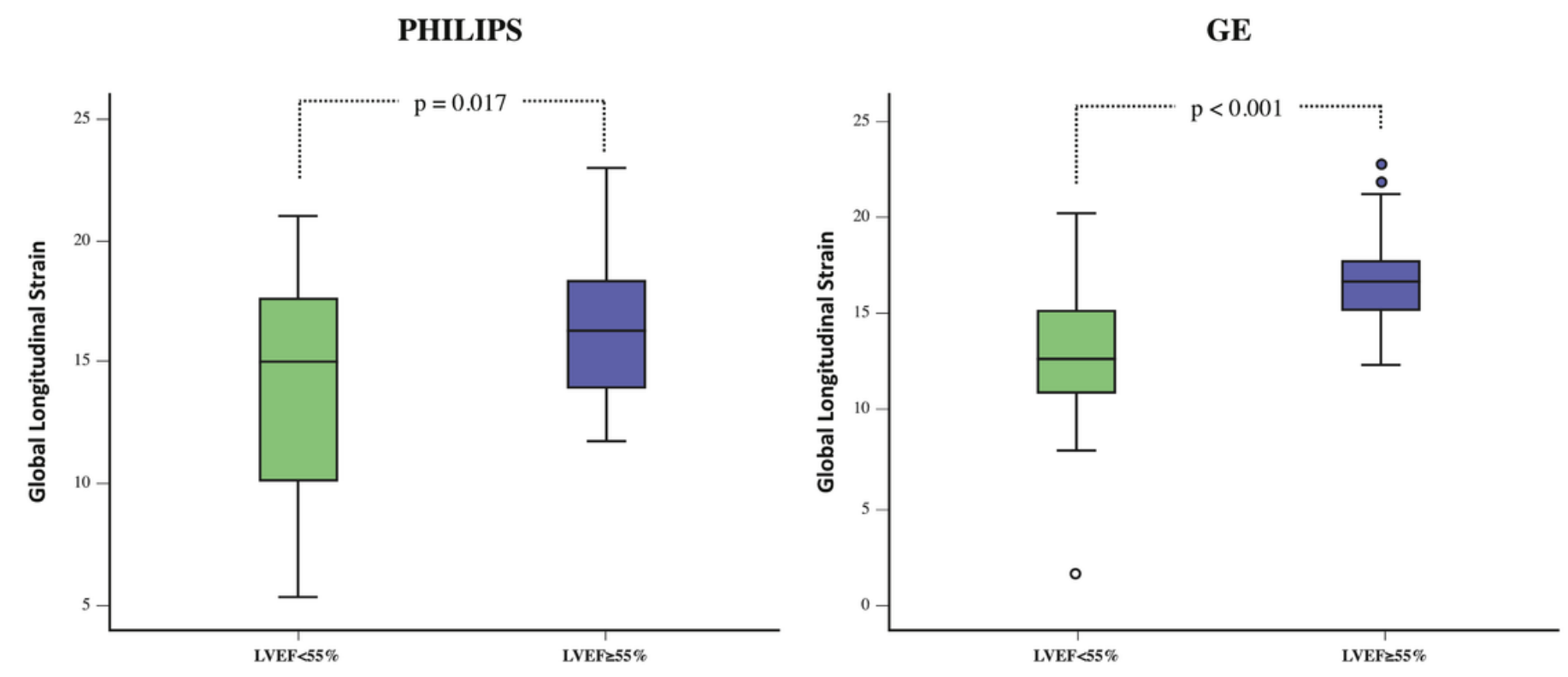

Figure 1

GLS values among patients with normal Vs. low LVEF, for the two vendors assessed. 


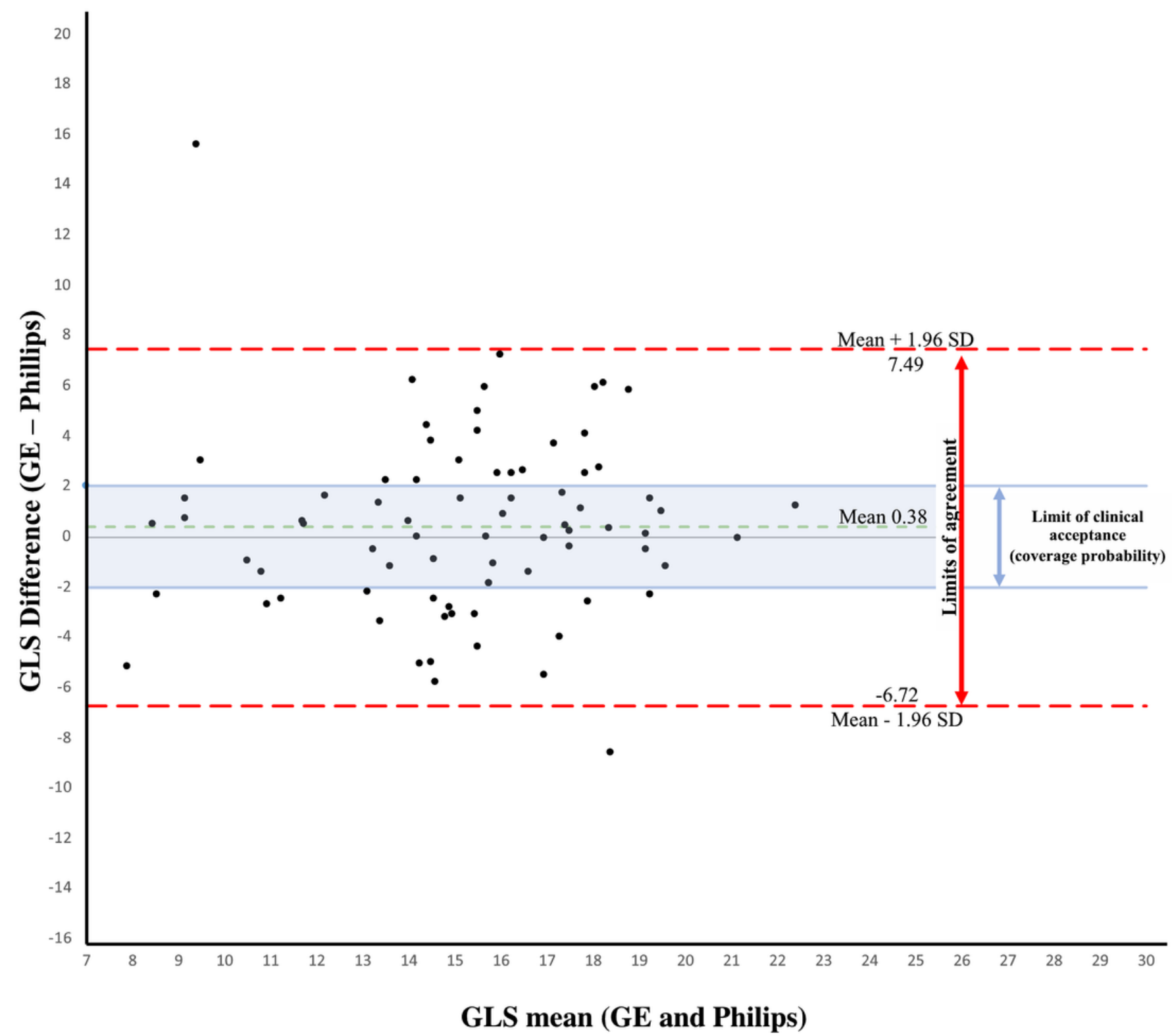

Figure 2

Bland-Altman plot showing inter-vendor variability of GLS values. The red dashed lines correspond to upper and lower LOA. The highlighted zone in blue corresponds to a pre-specified coverage probability of $2 \%$. 


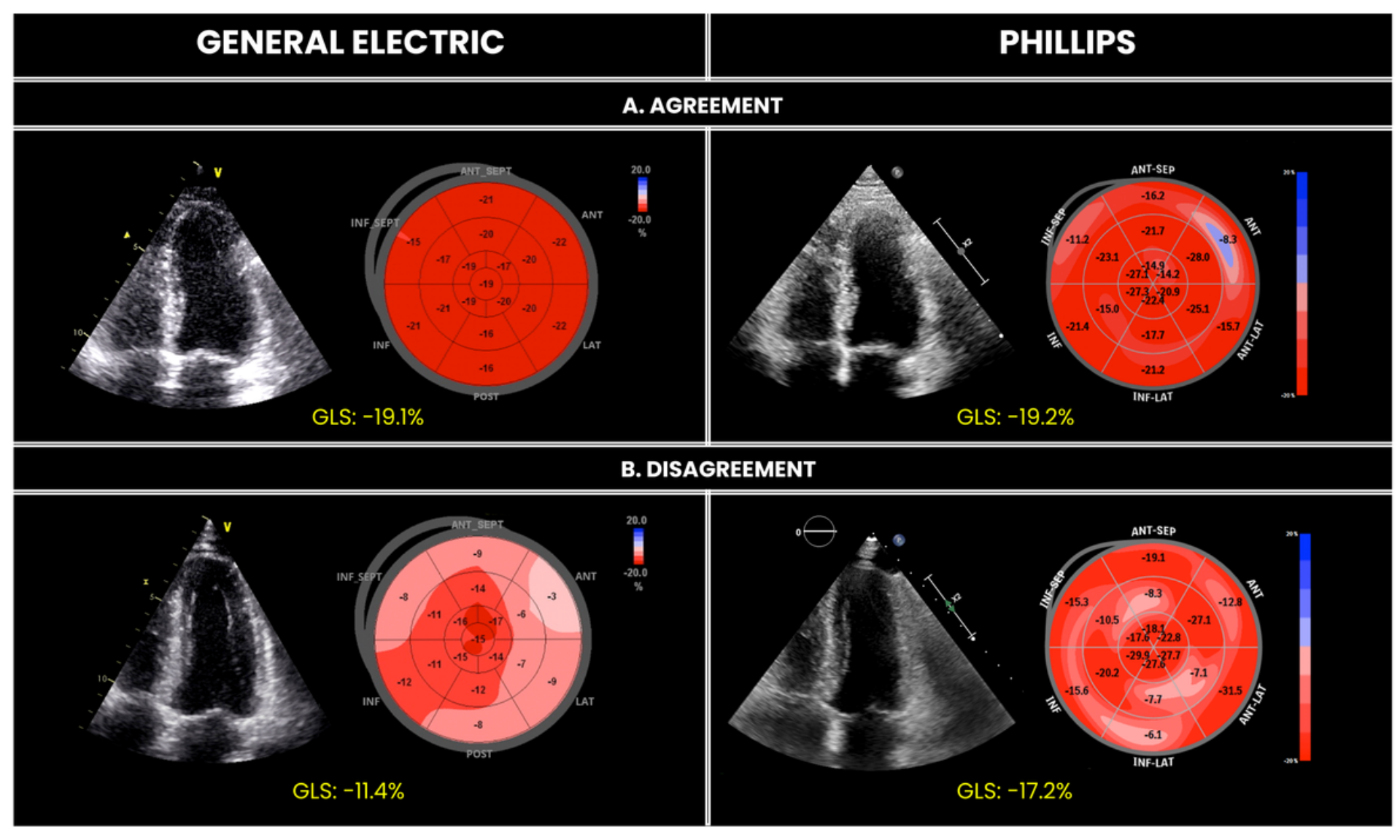

\section{Figure 3}

Simultaneous inter-vendor GLS analysis performed in two different HT patients. Panel A shows apical four-chamber frame and strain analysis a small inter-vendor GLS absolute difference of $0.1 \%$. Conversely, Panel B shows a wide inter-vendor GLS disagreement (absolute difference of 5,8\%). 


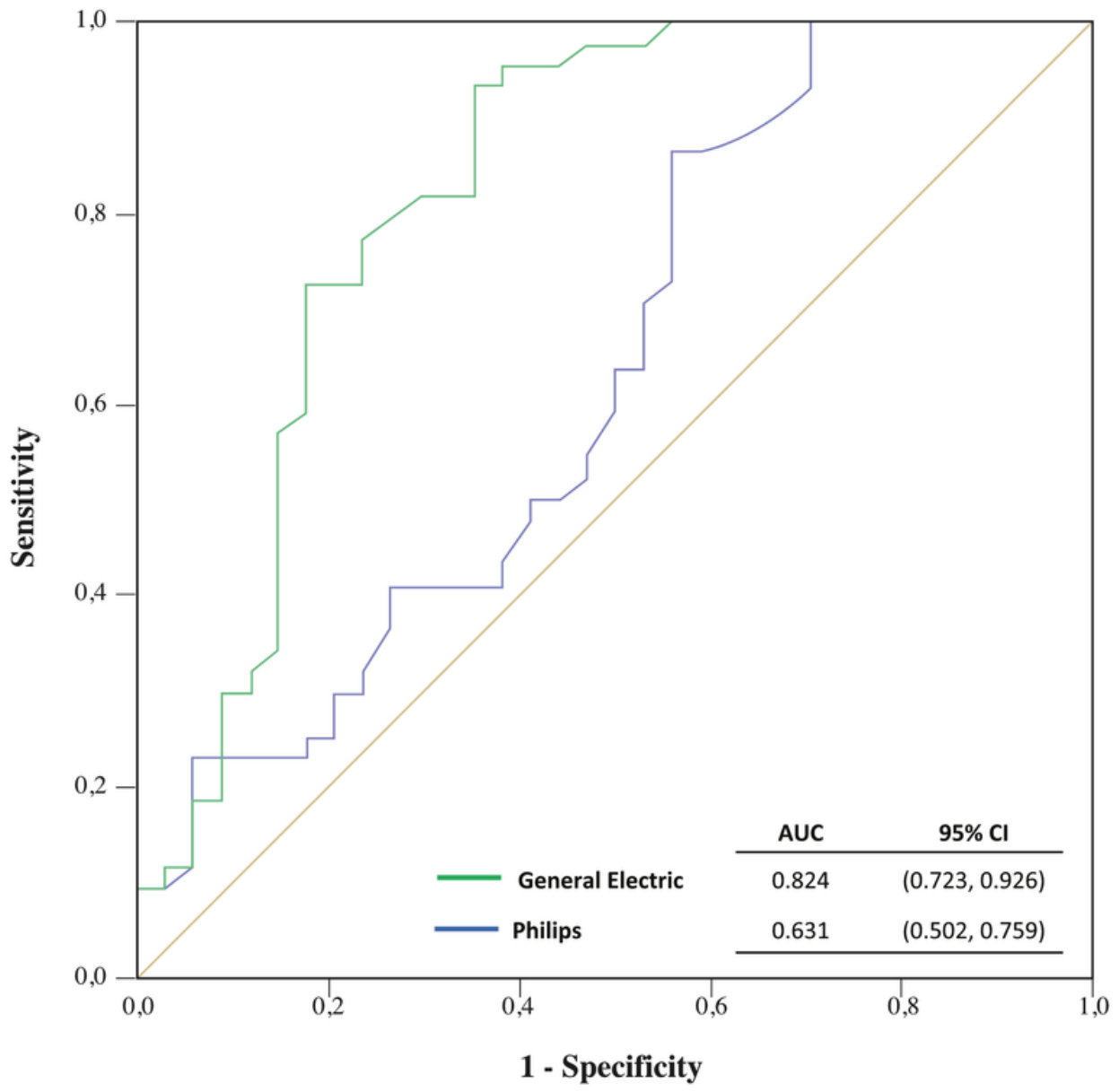

Figure 4

ROC curves and AUC of GLS to estimate a normal LVEF for both vendors.

\section{Supplementary Files}

This is a list of supplementary files associated with this preprint. Click to download.

- SupplementaryFigure1.tif

- SupplementaryFigure2.tif

- Supplementarytable1.docx 